\title{
A Paucity of Detection of the Middle East Respiratory Syndrome Coronavirus (MERS-CoV) Nucleic Acids in the Vaginal Secretions and the Urine of Some Naturally Infected Dromedary Camels
}

\author{
Maged Gomaa Hemida1,2*, Ali M. Ali ${ }^{3,4}$, Abdelmohsen Alnaeem ${ }^{5}$ \\ ${ }^{1}$ Department of Microbiology, College of Veterinary Medicine, King Faisal University, Al-Hasa, Saudi Arabia \\ ${ }^{2}$ Department of Virology, Faculty of Veterinary Medicine, Kafrelsheikh University, Kafrelsheikh, Egypt \\ ${ }^{3}$ Department of Biological Sciences, College of Science, King Faisal University, Al-Hasa, Saudi Arabia \\ ${ }^{4}$ Department of Botany and Microbiology, Faculty of Science, Minia University, Minia, Egypt \\ ${ }^{5}$ Department of Clinical Sciences, College of Veterinary Medicine, King Faisal University, Al-Hasa, Saudi Arabia \\ Email: *mhemida@kfu.edu.sa
}

How to cite this paper: Hemida, M.G., Ali, A.M. and Alnaeem, A. (2021) A Paucity of Detection of the Middle East Respiratory Syndrome Coronavirus (MERS-CoV) Nucleic Acids in the Vaginal Secretions and the Urine of Some Naturally Infected Dromedary Camels. Open Journal of Veterinary Medicine, 11, 327-335.

https://doi.org/10.4236/ojvm.2021.1112023

Received: December 4, 2021

Accepted: December 28, 2021

Published: December 31, 2021

Copyright $\odot 2021$ by author(s) and Scientific Research Publishing Inc. This work is licensed under the Creative Commons Attribution International License (CC BY 4.0).

http://creativecommons.org/licenses/by/4.0/

(c) (i) Open Access

\begin{abstract}
The dromedary camels play some critical roles in the evolution and transmission cycle of the MERS-CoV. There are no current available vaccines or antiviral medications to do prevention or to treat humans against the virus, respectively. Few studies reported the course of the MERS-CoV infection in dromedary camels under both the experiment and natural infection approaches. The curve of the viral particle, as well as its nucleic acids in nasal secretions of the dromedary camels, was reported in more details. However, MERS-CoV shedding in some other secretions such as vaginal secretions still understudied. The main goal of the current study was to check the possibility of MERS-CoV shedding of the MERS-CoV through the vaginal mucous membranes of some female camels. To achieve these goals, we conducted molecular surveillance on one dromedary camel herd in eastern Saudi Arabia. We collected the following swabs per each animal (nasal, rectal, and vaginal) as well as urine samples during the period from Jan 2020-April 2020. Testing of MERS-CoV nucleic acid (NA) was conducted in these swabs by the real-time PCR kits. Our results are showing that $(47 \%, 21 \%, 0 \%$, and $0 \%)$ of the (nasal, rectal, and vaginal) swabs, as well as the urine, were positive, respectively. All the tested vaginal swabs were negative. This may suggest the absence of MERS-CoV shedding in the female genital tract and urine samples despite the detection of the viral NA in the nasal swabs. This study is showing for the first time the
\end{abstract}


failure of detection of the MERS-CoV in the vaginal secretions of some naturally infected dromedary camels. This result is enriching our understanding of the MERS-CoV shedding in various body secretions and excretions.

\section{Keywords}

MERS-CoV, rt-RT-PCR, Dromedary Camels, Nasal Swabs, Rectal Swabs, Vaginal Swabs, Urine

\section{Introduction}

The MERS-CoV is one of the most important zoonotic coronaviruses candidates. The dromedary camels are still the only known reservoir for the virus [1] [2]. MERS-CoV infections in humans may range from a very mild form to severe fatal cases, which may lead to the death of the affected patients [3] [4]. Unfortunately, there are no licensed vaccines or antiviral medications for MERS-CoV until now. The other possible mitigations and containment approaches may include the adoption of some strategies based on the One Health approach [2]. This is in addition to the careful study of the viral replication cycle and pathogenesis especially the curve of the viral shedding in various body secretions and excretions of both humans and animals [3].

High seroprevalence of MERS-CoV was detected in dromedary camels in many countries especially Asia and Africa (ReF). The naïve dromedary camels under 2 years old are the most susceptible to MERS-CoV infection [5] [6] [7] [8]. Those young animals shed the virus in their body secretions particularly the respiratory tract at high levels during active infection [7] [9]. Some evidence of the animal to human transmission was reported earlier [10]. MERS-CoV infection in the dromedary camel is usually mild in the form of rhinorrhea and some lachrymation of the affected animals [11] [12]. The infectious MERS-CoV viral particles were detectable in the nasal tract for up to seven days after the experimental infection (dpi); however, the viral nucleic acids were detectable up to 35 dpi [11]. The viral shedding was also detected in both the nasal and rectal swabs of some dromedary camels [9]. Moreover, the viral nucleic acids were also detected in the seminal plasma of some male dromedary camels [13]. Some recent studies failed to detect MERS-CoV in the urine samples of some dromedary camels in Qatar [14]. The virus shedding was reported in some other viruses such as the Zika virus in the vaginal secretions for a prolonged period [15]. Meanwhile, several new studies reported the detection of the SARS-CoV-2 in the semen of some infected patients suggesting the potential sexual transmission during virus infection [16]. On the other hand, recent studies failed to detect the SARS-CoV-2 in the vaginal secretions of the severely affected females [17]. Breeding of dromedary camels under natural conditions usually involves a common male animal to inseminate several female camels in a particular region. Theoretically, if the common male animal was infected with MERS-CoV, it could trans- 
mit the virus to the other female animals through natural insemination. Reducing the exposure to the active MERS-CoV shedders will contribute substantially to the control of the virus. That is why mapping the viral shedding from dromedary camels through different body secretions and excretions including the urogenital tract (urine and vaginal secretions) could be very important to cut the transmission cycle of MERS-CoV. To our knowledge, shedding of the MERS-CoV in the vaginal fluids during the natural course of the MERS-CoV infection in both humans and animals is still not studied yet. We conducted the current study to check the possibility of shedding of MERS-CoV in the vaginal secretions of dromedary camels during active viral infection.

\section{Materials and Methods}

\subsection{The Dromedary Camel Herd}

This study was conducted in one dromedary camel herd in eastern Saudi Arabia. This herd consisted of 52 animals of various color coat-based breeds and all-female animals of variable age (including naive and old animals; 6 months up to 12 years old). This herd was reared in a station surrounded by a wire fence. The male animals are usually kept in a separate fence and allowed to approach the females (all live together), only during the breeding season and the process of mating between males and females (Figure 1(A)). During the regular sampling collection, at least $10 \%$ of the camel herd was randomly selected. Usually, we collected nasal, rectal, and vaginal swabs per each target animal. We also collected some urine samples per each visit, as described below.

\subsection{Animal Sampling}

We conducted molecular surveillance for the monitoring of MERS-CoV in one dromedary camel herd in eastern Saudi Arabia from January 2020 to April 2020.

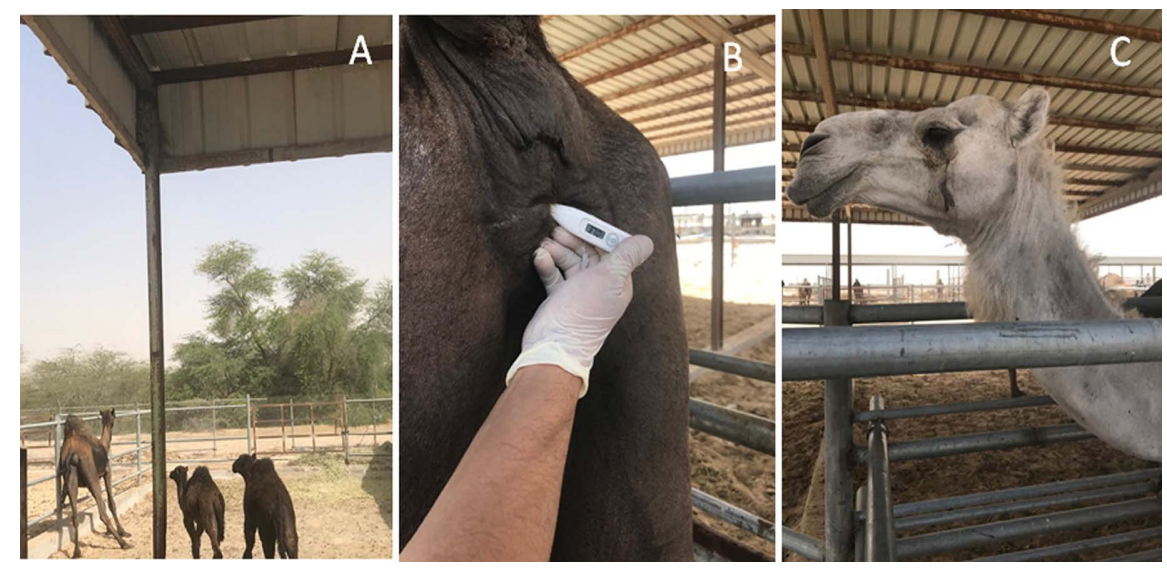

Figure 1. Some clinical signs of MERS-CoV naturally infected dromedary camels. (A): A picture of some dromedary camels living in the wire fence station. (B): Checking the temperature of some dromedary camel using a thermometer through the rectum. (C): A real-time PCR positive MERS-CoV dromedary camel showing excessive lacrimation and the tear stain the skin of the face. 
Samples were collected on a biweekly basis during the tenure of this study. We collected samples from at least nine animals per visit. We collected swabs (nasal, rectal, and vaginal) per animal. Urine was also collected during the active animal urination per each visit.

\subsection{Collection and Processing of the Nasal Swabs from Dromedary Camels Swabs (Nasal, Rectal, and Vaginal)}

We used sterile swabs to collect samples from the nasal passage, the rectum as previously described [7] [9]. The vaginal swabs were collected by widening the vulva of the animal by an assistant then introducing the swabs deep in the vagina to come in close contact with the inner mucous membranes of the vagina. These swabs were rotated several times inside the vaginal tract to allow the cotton pieces to absorption and carry some vaginal secretions. All the nasal, rectal and vaginal swabs were processed in the same manner. Each swab was transferred to $10 \mathrm{ml}$ sterile conical tubes then centrifuged for 5 minutes at $5000 \mathrm{RPM}$ at $4^{\circ} \mathrm{C}$. The supernatants were collected and transferred to fresh sterile tubes and stored at $\left(-20^{\circ} \mathrm{C}\right)$ for further testing.

\subsection{Collection and Processing of Urine Samples}

The urine samples were collected under complete aseptic conditions using sterile containers. While the animals attempt urination, the sterile containers were held in a vertical position to allow the urine to flow inside them. We collected at least $5 \mathrm{ml}$ per animal; then, the container was closed and placed on ice until transferred to the laboratory. The processing of the urine samples was done as previously described for the swabs above. About $1 \mathrm{ml}$ from the supernatants were stored at $\left(-20^{\circ} \mathrm{C}\right)$ for further testing.

\subsection{Extraction of the MERS-CoV-RNAs}

The total viral RNs were extracted from both the saliva and the conjunctival swabs obtained from our cross-sectional studies from dromedary camels by the Qiagen viral RNA (RNeasy Mini Kit, Qiagen, Hilden, Germany) extraction protocols. The procedure of the RNA extraction was carried out as per the instructions of the manufacturer (Qiagen GmbH, Hilden, Germany). The RNA concentration was measured immediately after the extraction by using the Nanodrop machine (Thermo Scientific NanoDrop 2000, Applied Biosystems, 850 Lincoln Centre Drive. Foster City, CA 94404, USA). The obtained MERS-CoVRNAs were kept at $\left(-80^{\circ} \mathrm{C}\right)$ until further testing.

\subsection{The Real-Time PCR Technique}

We used two specific MERS-CoV targets (Up-E and the ORF1a) real time-PCR kits for testing the collected saliva and conjunctival swabs. This procedure was conducted as previously described [7] [9] [13]. Samples were considered positive when their Ct values $\leq 29$. 


\section{Results}

\subsection{Results of the Clinical Examination of the Dromedary Camel Herd from January 2019-April 2020}

During our surveillance in the camel herd, we usually did a physical inspection of the animals before sample collection each time. During the tenure of this study, we identified some positive animals by the real-time PCR technique, as described above. We observed some vital signs of animals, especially the temperature, the respiratory rate, and the color of the mucous membranes, especially the vaginal membranes. The average body temperature of some positive MERS-CoV animals was $\left(37^{\circ} \mathrm{C} \pm 0.5^{\circ} \mathrm{C}\right.$ ) (Figure $1(\mathrm{~B})$ ). Our results are clearly showing a mild form of the respiratory signs, including some mild nasal discharges; some animals showed excessive lachrymation in which the tears of the animals stayed the ocular region around the eyes (Figure $1(C)$ ). The color of the vaginal mucus membranes was a bright rose normal color.

\subsection{Results of the Real-Time PCR Surveillance for the MERS-CoV in Dromedary Camel Herd January 2019-April 2020}

Our results of the molecular surveillance within this camel herd are showing that 47/105 of the nasal swabs and 21/105 rectal swabs were positive for the MERSCoV-NA (Table 1). However, all the tested vaginal swabs and urine samples were negative (Table 1).

\section{Discussion}

There are many unknown aspects of the molecular pathogenesis and transmission cycle of the MERS-CoV. The dromedary camels are still the known reservoir of the MERS-CoV up-to-date. However, the exact mechanisms of transmission from animals to humans are not well identified. High seroprevalence of MERS-CoV was detected in dromedary camels in the Arabian Peninsula and Africa [5] [8] [18]. Young animals under two years of age are more susceptible to viral infection; however, seroconversion of the old animals is more common in many studies [7] [9]. Special attention was paid to studying the curve of the MERS-CoV infection and its nucleic acids in the respiratory passage and nasal tract of the infected dromedary camels [3] [7] [9] [11]. MERS-CoV shedding was assessed by the detection of the infectious viral particles as well as the viral

Table 1. Results of eh Real-time PCR testing for the MERS-CoV swabs (nasal, rectal, vaginal) and urine of dromedary camels January 2020-April 2020.

\begin{tabular}{cccc}
\hline Batch/type of samples & Total tested & $(+\mathrm{Ve})$ & $(-\mathrm{Ve})$ \\
\hline Nasal swab & 105 & 47 & 58 \\
Rectal swab & 105 & 21 & 84 \\
Vaginal swab & 45 & 0 & 45 \\
Urine & 21 & 0 & 21 \\
\hline
\end{tabular}


nucleic acids (NA) in the nasal swabs under both the natural and experimental infection settings [7] [9] [10] [11] [12]. The current study also reported the detection of the viral nucleic acid in the nasal swabs during the tenure of this study (Table 1). Earlier studies reported that $97.6 \%$ of tested nasal swabs from animals admitted to slaughtering in Qatar were positive for MERS-CoV [19]. These findings are very much consistent with our results since $47 \%$ of the tested nasal swabs were positive for the MERS-CoV-NA. Thus, detection of the MERS-CoV and/or its nucleic acids in the nasal passage is considered the gold standard for the diagnosis of MERS-CoV in both humans and dromedary camels. Detection of the nucleic acids of the MERS-CoV in the rectal swabs of dromedary camels was also reported in some earlier studies [7] [9] [19]. However, the detection rate of MERS-CoV in the rectal swabs was less than that in the nasal swabs [7] [9] [19]. Our results are also confirming these findings. About $20 \%$ of the tested rectal swabs in this study showed positive reactions to the MERS-CoV-NA (Table 1). Some studies reported the absence of the MERS-CoV shedding in the urine of infected camels [14]. However, the possibility of shedding of the virus in other body secretions is still further investigated. Currently, there is a lack of any effective licensed vaccine or medications available in the market to prevent or cure the viral infection, respectively. Understanding the viral shedding in various body secretions of dromedary camels will help us not only in understanding the dynamics of the viral spread within a certain population of animals but also will help in the mitigation of the virus transmission to humans. Detection of the MERS-CoV in the seminal fluids of some male dromedary camels is recently reported [13]. The outcomes of this study highlighted the potential roles of male animals in the transmission of the virus; however, large-scale studies are required to confirm these studies. However, shedding of the MERS-CoV in other body organs and the female genital tract was not studied yet. We designed this study to mainly check the possibility of MERS-CoV shedding in the vaginal tract of some MERS-CoV infected female camels. Looking at the viral shedding in the urine as well as rectal swabs of these animals was another objective. We identified a positive MERS-CoV dromedary camel herd during early 2019 from January-April. We were not able to detect the MERS-CoV-NA in the vaginal secretions of the female camels during the duration of this study (Table 1). Our results are very much consistent with some recent data from SARS-CoV-2, which are showing the absence of the SARS-CoV-2 in the vaginal secretion of the several affected women during the active course of infection [16] [17]. This result is in contrast to the detection of the viral nucleic acids in some seminal plasma of male camels recently reported [13]. Meanwhile, recent studies showed the detection of the SARS-CoV-2 in the semen of some infected male patients, which affects the semen quality in some cases [20]. This was in contrast to some studies that showed the absence of SARS-CoV-2 in the semen of some recovered patients [21]. However, all these studies required some large-scale control studies to confirm the possibility of the sexual transmission of some coronavirus candi- 
dates such as MERS-CoV and SARS-CoV-2. The urine of the dromedary camels may be used as a medication to cure some important diseases such as diabetes mellitus based on some cultural backgrounds in the Middle East and Africa [3] [22]. An earlier study reported the absence of MERS-CoV shedding in the urine of some dromedary camels in Qatar [14]. This finding is very much consistent with our results, suggesting the absence of MERS-CoV shedding in the urine of positive MERS-CoV infected animals. The high alkalinity of the dromedary camel urine (ranges from 8.2 to 9.2) may have some deleterious effects on the MERS-CoV-NA [3] [23]. On the other hand, the lack of detection of MERSCoV-NA in the vaginal secretions of some positive animals could be attributed to the absence of the viral receptors in the genital tract of female dromedary camels, the $\mathrm{PH}$ of the vaginal tract may be deleterious of the viral growth and multiplication, the urine passing through the vaginal tract may have some negative impacts on the viral nucleic acids. In conclusion, our study showed, for the first time, the absence of the detection of MERS-CoV nucleic acids in the vaginal swabs of some naturally infected female camels. Meanwhile, it also confirmed that the nasal swabs consider the sample of choice in the diagnosis of MERS-CoV when compared to other samples, particularly the rectal swabs. Furthermore, it also confirmed the absence of the MERS-CoV detection in the urine samples of the naturally infected dromedary camels. A large-scale cohort study is required for a better understanding of the dynamics of the MERS-CoV shedding in various body secretions and execrations.

\section{Statement of Ethics}

All handling and animal experiments carried out in this study were conducted as per the instructions of the Animal Ethics protocols and the National Committee of Bio-Ethics, King Abdul-Aziz City of Science and Technology, Royal Decree No. M/59.

\section{Data Availability Statement}

Data is available upon request.

\section{Author Contributions}

MGH and AAA; designed experiments, fieldwork, laboratory processing and techniques, data interpretations, writing the manuscript. MA; laboratory experiments, data analysis, wrote the manuscript. MAA; data analysis, wrote the manuscript.

\section{Funding Sources}

We wish to thank the King Abdul-Aziz City for Science and Technology (KACST), Saudi Arabia, for their generous funding through the MERS-CoV research grant program (number 24/1: 20-0004), which is a part of the Targeted Research Program (TRP). 


\section{Conflict of Interest}

All authors declare no conflict of interest.

\section{References}

[1] Hemida, M.G. (2019) Middle East Respiratory Syndrome Coronavirus and the One Health Concept. PeerJ, 7, e7556. https://doi.org/10.7717/peerj.7556

[2] Hemida, M.G. and A. Alnaeem (2019) Some One Health Based Control Strategies for the Middle East Respiratory Syndrome Coronavirus. One Health, 8, Article ID: 100102. https://doi.org/10.1016/j.onehlt.2019.100102

[3] Hemida, M.G., Elmoslemany, A., Al-Hizab, F., Alnaeem, A., Almathen, F., Faye, B., et al. (2017) Dromedary Camels and the Transmission of Middle East Respiratory Syndrome Coronavirus (MERS-CoV). Transboundary and Emerging Diseases, 64, 344-353. https://doi.org/10.1111/tbed.12401

[4] Zumla, A., Hui, D.S. and Perlman, S. (2015) Middle East Respiratory Syndrome. Lancet, 386, 995-1007. https://doi.org/10.1016/S0140-6736(15)60454-8

[5] Chu, D.K., Oladipo, J.O., Perera, R.A., Kuranga, S.A., Chan, S.M., Poon, L.L., et al. (2015) Middle East Respiratory Syndrome Coronavirus (MERS-CoV) in Dromedary Camels in Nigeria, 2015. EuroSurveill, 20, Article ID: 30086. https://doi.org/10.2807/1560-7917.ES.2015.20.49.30086

[6] Chu, D.K.W., Hui, K.P.Y., Perera, R.A.P.M., Miguel, E., Niemeyer, D., Zhao, J., et al. (2018) MERS Coronaviruses from Camels in Africa Exhibit Region-Dependent Genetic Diversity. Proceedings of the National Academy of Sciences of the United States of America, 115, 3144-3149. https://doi.org/10.1073/pnas.1718769115

[7] Hemida, M.G., Chu, D., Poon, L., Perera, R., Alhammadi, M.A., Ng, H., et al. (2014) MERS Coronavirus in Dromedary Camel Herd, Saudi Arabia. Emerging Infectious Diseases, 20, 1231-1234. https://doi.org/10.3201/eid2007.140571

[8] Hemida, M.G., Perera, R.A., Al Jassim, R.A., Kayali, G., Siu, L.Y., Wang, P., et al. (2014) Seroepidemiology of Middle East Respiratory Syndrome (MERS) Coronavirus in Saudi Arabia (1993) and Australia (2014) and Characterisation of Assay Specificity. EuroSurveill, 19, Article ID: 20828. https://doi.org/10.2807/1560-7917.ES2014.19.23.20828

[9] Hemida, M.G., Alnaeem, A., Chu, D.K., Perera, R.A., Chan, S.M., Almathen, F., et al. (2017) Longitudinal Study of Middle East Respiratory Syndrome Coronavirus Infection in Dromedary Camel Herds in Saudi Arabia, 2014-2015. Emerging Microbes \& Infections, 6, e56. https://doi.org/10.1038/emi.2017.44

[10] Azhar, E.I., El-Kafrawy, S.A., Farraj, S.A., Hassan, A.M., Al-Saeed, M.S., Hashem, A.M., et al. (2014) Evidence for Camel-to-Human Transmission of MERS Coronavirus. New England Journal of Medicine 370, 2499-2505. https://doi.org/10.1056/NEJMoa1401505

[11] Adney, D.R., van Doremalen, N., Brown, V.R., Bushmaker, T., Scott, D., de Wit, E., et al. (2014) Replication and Shedding of MERS-CoV in Upper Respiratory Tract of Inoculated Dromedary Camels. Emerging Infectious Diseases, 20, 1999-2005. https://doi.org/10.3201/eid2012.141280

[12] Alnaeem, A., Samy Kasem, Ibrahim Qasim, Ali Al-Doweriej, Ali Al-Houfufi Abdulatif Alwazan, et al. (2020) Some Pathological Observations on the Naturally Infected Dromedary Camels (Camelus dromedarius) with the Middle East Respiratory Syndrome Coronavirus (MERS-CoV) in Saudi Arabia 2018-2019. Veterinary Quarterly, 40, 190-197. https://doi.org/10.1080/01652176.2020.1781350 
[13] Hemida, M.G., Waheed, M. Ali, A.M. and Alnaeem, A. (2020) Detection of the Middle East Respiratory Syndrome Coronavirus in Dromedary Camel's Seminal Plasma in Saudi Arabia 2015-2017. Transboundary and Emerging Diseases, 67, 2609-2614. https://doi.org/10.1111/tbed.13610

[14] Farag, E.A., Haagmans, B.L., Al-Romaihi, H., Mohran, K., Haroun, M., El-Sayed, A.M., et al. (2019) Failure to Detect MERS-CoV RNA in Urine of Naturally Infected Dromedary Camels. Zoonoses and Public Health, 66, 437-438.

https://doi.org/10.1111/zph.12583

[15] Murray, K.O., Gorchakov, R., Carlson, A.R., Berry, R., Lai, L., Natrajan, M., et al. (2017) Prolonged Detection of Zika Virus in Vaginal Secretions and Whole Blood. Emerging Infectious Diseases, 23, 99-101. https://doi.org/10.3201/eid2301.161394

[16] Tatu, A.L., Nadasdy, T. and Nwabudike, L.C. (2020) Observations about Sexual and other Routes of SARS-CoV-2 (COVID-19) Transmission and Its Prevention. Clinical and Experimental Dermatology, 45, 761-762. https://doi.org/10.1111/ced.14274

[17] Qiu, L., Liu, X., Xiao, M., Xie, J., Cao, W., Liu, Z., et al. (2020) SARS-CoV-2 Is Not Detectable in the Vaginal Fluid of Women with Severe COVID-19 Infection. Clinical Infectious Diseases, 71, 813-817. https://doi.org/10.1093/cid/ciaa375

[18] Hemida, M.G., Perera, R.A., Wang, P., Alhammadi, M.A., Siu, L.Y., Li, M., et al. (2013) Middle East Respiratory Syndrome (MERS) coronavirus Seroprevalence in Domestic Livestock in Saudi Arabia, 2010 to 2013. EuroSurveill, 18, Article ID: 20659. https://doi.org/10.2807/1560-7917.ES2013.18.50.20659

[19] Mohran, K.A., Farag, E.A., Reusken, C.B., Raj, V.S, Lamers MM, Pas SD, et al. (2016) The Sample of Choice for Detecting Middle East Respiratory Syndrome Coronavirus in Asymptomatic Dromedary Camels Using Real-Time Reversetranscription Polymerase Chain Reaction. Revue scientifique et technique, 35, 905-911. https://doi.org/10.20506/rst.35.3.2578

[20] Perry, M.J., Arrington, S., Neumann, L.M., Carrell, D. and Mores, C.N. (2020) It Is Currently Unknown Whether SARS-CoV-2 Is Viable in Semen or Whether COVID-19 Damages Spermatozoa. Andrology, 9, 30-32. https://doi.org/10.1111/andr.12831

[21] Pan, F., Xiao, X., Guo, J., Song, Y., Li, H., Patel, D.P., et al. (2020) No Evidence of Severe Acute Respiratory Syndrome-Coronavirus 2 in Semen of Males Recovering from Coronavirus Disease 2019. Fertility and Sterility, 113, 1135-1139.

https://doi.org/10.1016/j.fertnstert.2020.04.024

[22] Gossner, C., Danielson, N., Gervelmeyer, A., Berthe, F., Faye, B., Kaasik Aaslav, K., et al. (2016) Human-Dromedary Camel Interactions and the Risk of Acquiring Zoonotic Middle East Respiratory Syndrome Coronavirus Infection. Zoonoses and Public Health, 63, 1-9. https://doi.org/10.1111/zph.12171

[23] Ali, A., Baby, B. and Vijayan, R. (2019) From Desert to Medicine: A Review of Camel Genomics and Therapeutic Products. Frontiers in Genetics, 10, Article No. 17. https://doi.org/10.3389/fgene.2019.00017 